\title{
Extreme All-dielectric Huygens' Metasurfaces based on Quasi-bound States in the Continuum
}

\author{
Mingkai Liu ${ }^{1}$, Duk-Yong Choi ${ }^{2,3}$ \\ ${ }^{I}$ Nonlinear Physics Center, Research School of Physics and Engineering, the Australian National University, Canberra, ACT 2601, Australia \\ ${ }^{2}$ Laser Physics Center, Research School of Physics and Engineering, the Australian National University, Canberra, ACT 2601, Australia \\ ${ }^{3}$ College of Information Science and Technology, Jinan University, Guangzhou, Guangdong 510632, China \\ Mingkai.liu@anu.edu.au
}

\begin{abstract}
We introduce and experimentally demonstrate a novel platform to connect the concepts of Huygens' condition and bound states in the continuum, allowing Huygens' metasurfaces with controllable Q-factors over orders of magnitude. ( 2019 The Author(s) OCIS codes: $260.0260,160.3918$
\end{abstract}

Recent progress in Huygens' metasurfaces demonstrated unprecedented capability to manipulate electromagnetic waves in a highly efficient fashion using ultra-compact platforms. The underlying principle is impedance matching of the metasurfaces and the environment by overlapping and balancing resonances that have different parities $[1,2]$. High index dielectric materials have been employed to minimize the absorption loss at optical frequencies [3]; however, all the proposed and realized dielectric Huygens' metasurfaces are relatively broadband due to the strong radiative loss. This broadband feature is useful in many application [4], but it also brings in great challenges when creating tunable metadevices with a large dynamic range, or nonlinear optical devices that require high nonlinear efficiency, or metasurfaces that can control the chromatic dispersion of pulses. Importantly, the quality factors (Qfactor) of the resonances in current Huygens' metasurfaces are complicated to tune without breaking the delicate balance for the Huygens' condition. It remains an open question whether it is possible to maintain Huygens' condition while controlling the Q-factors of the resonances.

Here we show a generic approach to achieve Huygens' metasurfaces with controllable Q-factors, and in the extreme case, the Q-factors can approach infinity [5]. This novel feature is directly linked to the concept of bound states in the continuum (BICs) [6]. A typical feature of BICs in resonant systems is that the Q-factor can approach infinity asymptotically (at least theoretically) when the parameter of the resonator approaches a critical point. This phenomenon occurs when the total overlapping of the resonant modes and the radiative channels approaches zero. In practice, the Q-factor is always finite due to various imperfection in fabrication, and therefore any observable BIC is quasi-BIC. Unlike conventional quasi-BICs that are manifested as sharp amplitude modulation, the quasi-BICs operated under the Huygens' condition are manifested as highly dispersive pure phase modulation over a $2 \pi$ range. This novel phenomenon occurs when two quasi-BICs with matched Q-factors but opposite mode parities are spectrally overlapped. Although many studies of high-Q metasurfaces have been performed, nearly all studied platforms rely on the nearfield interaction of so-called bright modes and dark modes. This requirement inevitably introduce additional resonant background in the transmission spectrum. In contrast, our novel approach do not rely on the nearfield coupling of bright modes and dark modes, and it allows a direct tuning of the radiative decay rates of the quasi-BICs while maintaining a clean transmission background.

(a)

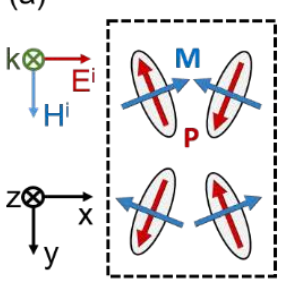

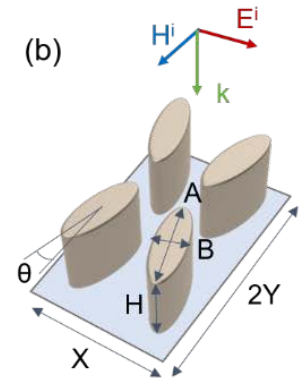
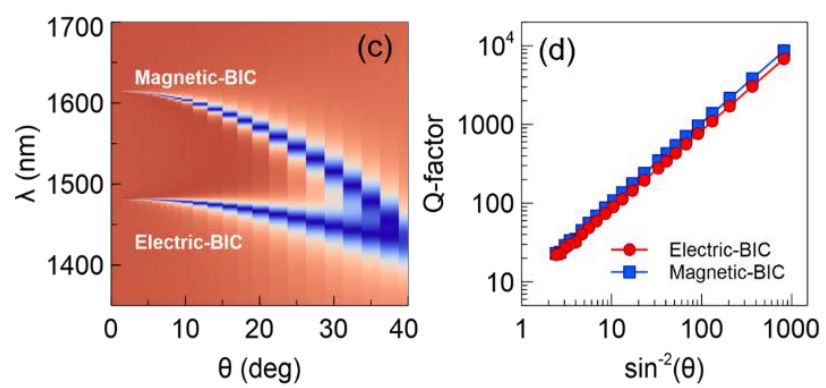

Fig. 1. (a) Schematic of the concept of Huygens' quasi-BICs. (b) Design based on zig-zag array of anisotropic silicon meta-atoms. (c) Simulated transmission spectra of the electric and magnetic quasi-BICs as a function of angle $\theta$, with $X=900, Y=600, H=540, A=530, B=170$, (units in $\mathrm{nm}$ ). (d) the corresponding Q-factors.

To realize this novel effect, we introduce an all-dielectric metasurface composed of zig-zag arrays of anisotropic meta-atoms. This structure was first introduced for realizing optomechanically-induced chirality [7], and has been 
recently adapted for bio-sensing due to its high-Q factor and strong field localization. The quasi-BICs demonstrated in our studied are collective resonances built on the Mie-like modes of the meta-atoms, with electric dipole and magnetic dipole moments being the dominant contribution. Therefore, we dub the two collective resonances as electric quasi-BIC and magnetic quasi-BIC. One unique feature of the zig-zag structure is that the dominant dipole moments of the meta-atoms $\left(\mathrm{p}_{\mathrm{y}}, \mathrm{m}_{\mathrm{x}}\right)$ are perpendicular to the incident polarizations $\left(\mathrm{E}_{\mathrm{x}}, \mathrm{H}_{\mathrm{y}}\right)$ (see Fig. 1a); however, due to antisymmetric distribution of the $\left(\mathrm{p}_{\mathrm{y}}, \mathrm{m}_{\mathrm{x}}\right)$ components within each unit-cell, their net overlapping with the polarizations $\left(\mathrm{E}_{\mathrm{y}}, \mathrm{H}_{\mathrm{x}}\right)$ is zero and thus their out-coupling is forbidden by symmetry. Only the small $\left(\mathrm{p}_{\mathrm{x}}, \mathrm{m}_{\mathrm{y}}\right)$ components are nonzero and contribute to the radiative loss. By changing the geometries of the meta-atoms, such as their orientation angles $\theta$, the Q-factors of the resonances can be controlled deterministically.

We develop a theoretical model and show analytically that the Q-factors of the electric and magnetic quasi-BICs follow the same scaling factor, i.e. $Q_{E} \propto \sin ^{-2} \theta, Q_{M} \propto \sin ^{-2} \theta$, both of which grow asymptotically as $\theta$ approaches zero and become infinity when $\theta=0$. This is a direct manifestation of BICs. This behavior is confirmed by numerical simulation. To mimic the experimental condition, we simulate Huygens' metasurfaces based on hydrogenated amorphous silicon (a-Si:H) meta-atoms embedded in a homogeneous background of Poly(methylmethacrylate) (PMMA) (see Fig. 1b). The asymptotic behavior of the Q-factors are confirmed, as shown in Fig. 1c and 1d. One unique feature of this paradigm is that since both $Q_{E}$ and $Q_{M}$ follow the same scaling factor, as long as the Q-factors are matched for a particular design, they would remain matching over a wide range of $Q$ as $\theta$ decreases (see Fig. 1d).
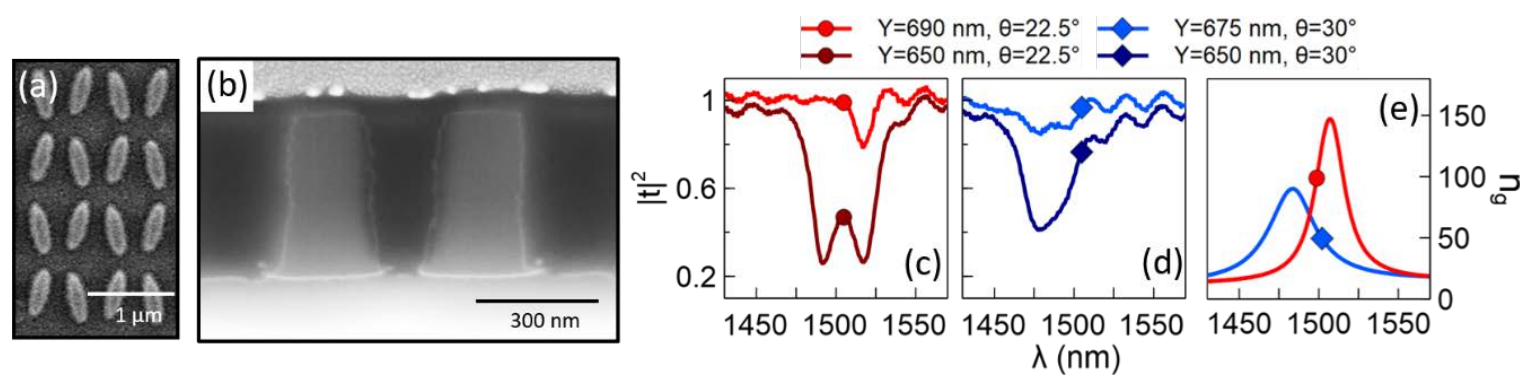

Fig. 2. (a) and (b) The SEM images of the fabricated metasurfaces. The post-fabrication measured geometries: $\mathrm{X}=900, \mathrm{~A}=530, \mathrm{~B}=199, \mathrm{H}=520$ (units in $\mathrm{nm}$ ). (c) and (d) measured transmission spectra for different designs. (e) The corresponding simulated group indexes at the crossing point of two quasi-BICs.

To verify the concept, we fabricated Huygens' metasurfaces based on a-Si:H, using electron-beam lithography and fluorine-based inductively coupled plasma-reactive ion etching (see SEM images in Fig. 2a and 2b). To minimize the bianisotropy introduced by the glass substrate, a layer of PMMA was spin-coated to cover the metasurface. Two designs of samples were fabricated, with $\theta=22.5^{\circ}$ and $30^{\circ}$ being the only difference in parameters. Due to fabrication limitation, the chosen parameters is a trade-off between the Q-factors and the transmission amplitude at the crossing point. The measured linewidths of the quasi-BICs are around $20 \mathrm{~nm}(\mathrm{Q} \sim 75)$ and $30 \mathrm{~nm}(\mathrm{Q} \sim 50)$, with the transmission increases to around $80 \%$ and $85 \%$ at the crossing points for designs $\theta=22.5^{\circ}$ and $\theta=30^{\circ}$, respectively (see Fig. 2c and $2 \mathrm{~d}$ ). The corresponding group indexes shown in Fig. 2e further confirm that the dispersion of the Huygens' metasurface can be fine-tuned.

To conclude, we proposed and demonstrated the concept of extreme Huygens' metasurfaces both theoretically and experimentally. This approach bridges the concepts of Huygens' condition and optical bound-states in the continuum, pointing a practical route to control the dispersion of Huygens' metasurfaces and to realize highefficiency nanophotonic devices that are highly dispersive and spectrally selective.

[1] C. Pfeiffer, A. Grbic, “Metamaterial Huygens' Surfaces: Tailoring Wave Fronts with Reflectionless Sheets”, Phys. Rev. Lett. 110, 197401 (2013).

[2] M. Liu, Y. Zhang, X. Wang, C. Jin, “Incident-angle-insensitive and polarization independent polarization rotator”, Optics Express 18, 1199012001, (2010)

[3] M. Decker, I. Staude, M. Falkner, J. Dominguez, D. N. Neshev, I. Brener, T. Pertsch, Y. S. Kivshar, "High-efficiency dielectric Huygens' surfaces". Adv. Opt. Mater. 3, 813-20, (2015).

[4] S. Kruk, B. Hopkins, I. I. Kravchenko, A. Miroshnichenko, D. N. Neshev, Y. S. Kivshar, "Broadband highly efficient dielectric metadevices for polarization control". APL Photonics. 1, 030801, (2016).

[5] M. Liu, D. Choi, "Extreme Huygens' metasurfaces based on quasi-bound states in the continuum", Nano Lett. (accepted) (2018)

[6] C-W. Hsu, B. Zhen, A. D. Stone, J. D. Joannopoulos, M. Soljačić, "Bound states in the continuum”, Nature Rev. Mater. 1, 16048, (2016).

[7] M. Liu, D. A. Powell, R. Guo, I. V. Shadrivov, and Y. S. Kivshar, "Polarization-induced chirality in metamaterials via optomechanical interaction”, Adv. Opt. Mater., 5, 1600760, (2017) 